\title{
Assistência a saúde prestada a hipertensos e diabéticos no Brasil: revisão integrativa
}

\author{
Health care provided to hypertensive and diabetic patients in Brazil: an integrative review \\ Atención médica proporcionada a pacientes hipertensos y diabéticos en Brasil: una \\ revisión integradora
}

Fábio da Costa Ferreira ${ }^{1 *}$, Marcos Valério Santos da Silva1.

\begin{abstract}
RESUMO
Objetivo: Analisar as evidências disponíveis na literatura cientifica sobre a assistência à saúde prestada aos usuários hipertensos e diabéticos no Brasil. Métodos: Trata-se de estudo de revisão integrativa das bases de dados Scientific Eletronic Library Online (Scielo), Literatura Latino-Americana e do Caribe (LILACS) e Base de Dados em Enfermagem (BDENF), utilizando-se descritores de acordo com os Descritores em Ciências da Saúde (DeCS) e combinando-os por meio do uso do operador booleano AND, critérios de inclusão: publicações de 2009 a 2018 em português, espanho e inglês, sendo excluídos os duplicados e aqueles que não aboradaram a temática. Resultados: Foram selecionados nove estudos para analise, os quais abordaram a temática proposta pelo atual estudo, logo emergiram dois principais grupos temáticos: Estrutura das unidades de saúde e Processos de atendimentos. Tendo os mesmos impactos relevantes sobre a assistencia prestadas aos usuários hipertensos e diabéticos. Considerações finais: Como limitação destacase a inclusão de inúmeros estudos com diferentes delineamentos de pesquisa dificultando a analise, quanto potencialidade tem a amostragem dos estudos selecionados, pois proporcionou um efetivo entendimento da qualidade da assitencia prestada aos hipertensos e diabéticos no Brasil. Além disso, os achados subsidiarão a atuação de gestores e profissionais de saúde.
\end{abstract}

Palavras-chave: Assistência em saúde, Hipertensão, Diabetes mellitus.

\begin{abstract}
Objective: To analyze the evidence available in the scientific literature on health care provided to hypertensive and diabetic users in Brazil. Methods: This is a integrative review study of the Scientific Electronic Electronic Library Online (SciELO), Latin American and Caribbean Literature (LILACS) and Nursing Database (BDENF) databases, using descriptors according to the Health Sciences Descriptors (DeCS) and combining them using the Boolean operator AND, inclusion criteria: publications from 2009 to 2018 in Portuguese, Spanish and English, with duplicates and those that did not address the topic being excluded. Results: Nine studies were selected for analysis, which addressed the theme proposed by the current study, soon two main thematic groups emerged: Structure of health units and Care processes. Having the same relevant impacts on the assistance provided to hypertensive and diabetic users. Final considerations: As a limitation, the inclusion of countless studies with different research designs stands out, making the analysis difficult, how much potential has the sampling of the selected studies, as it provided an effective understanding of the quality of the assistance provided to hypertensive and diabetic patients in Brazil. In addition, the findings will support the work of managers and health professionals.
\end{abstract}

Key words: Health care, Hypertension, Diabetes mellitus.

\section{RESUMEN}

Objetivo: Analizar la evidencia disponible en la literatura científica sobre la atención de salud brindada a usuarios hipertensos y diabéticos en Brasil. Métodos: Se trata de un estudio de revisión integradora de las bases de datos de la Biblioteca Electrónica Electrónica Científica en Línea (SciELO), Literatura Latinoamericana y del Caribe (LILACS) y Base de Datos de Enfermería (BDENF), utilizando descriptores según los Descriptores de Ciencias de la Salud (DeCS) y combinándolos el operador booleano AND, criterios

1 Universidade Federal do Pará (UFPA), Belém - PA. *E-mail: ferreirafabio582@gmail.com 
de inclusión: publicaciones de 2009 a 2018 en portugués, español e inglés, con duplicados y aquellas que no abordaron el tema excluido. Resultados: Se seleccionaron nueve estudios para su análisis, los cuales abordaron la temática propuesta por el presente estudio, pronto surgieron dos grandes grupos temáticos: Estructura de las unidades de salud y Procesos de atención. Tener los mismos impactos relevantes en la asistencia brindada a usuarios hipertensos y diabéticos. Consideraciones finales: Como limitación, se destaca la inclusión de numerosos estudios con diferentes diseños de investigación, dificultando el análisis, cuánto potencial tiene el muestreo de los estudios seleccionados, ya que brindó una comprensión efectiva de la calidad de la atención brindada a hipertensos y diabéticos. Pacientes en Brasil. Además, los hallazgos respaldarán el desempeño de los administradores y profesionales de la salud.

Palabras clave: Salud, Hipertensión, Diabetes mellitus.

\section{INTRODUÇÃO}

A Hipertensão Arterial Sistêmica (HAS) e o Diabetes Mellitus (DM), caracterizam-se atualmente entre as condições crônicas mais prevalentes. Juntas, são destacadas como as mais terríveis causas primárias de doenças renais e cardiovasculares no panorama mundial, e culpadas por 14,4 milhões de mortes no mundo, das quais 9,4 milhões ocasionadas por complicações dos níveis pressóricos elevados e 5,0 milhões atribuíveis ao desequilíbrio metabólico (HU JR CJ, 2017; TAO L, et al., 2015).

As Doenças Crônicas não Transmissiveis (DCNT), como as doenas cardiovasculares que equivalem cerca de $63 \%$ de todas as causas de morte na população mundial. No Brasil não é diferente, dados coletados em 2009 apresentados pelo Ministério da Saúde (MS) mostram que as doenças cardiovasculares são as maiores causas de óbito no país. A prevenção é a melhor forma de controlar os fatores de risco e consequentemente reduzir as taxas de mortalidade (FIGUEIREDO JR AM, et al., 2019).

Essa conjuntura é preocupante e impõem a necessidade de investimentos em melhorias na qualidade do manejo dessas morbidades, e de maneira especial na Atenção Primária à Saúde (APS), por ser o nível de atenção responsável pela detecção das condições crônicas e acompanhamento daqueles já diagnosticados, evitando suas complicações e necessidade de internação hospitalar (SILVA LB, 2018).

Diante desse contexto, torna-se mais difícil para o sistema fornecer ao usuário o cuidado teoricamente mais eficiente e acessível no mercado, devido as pressões impostas sobre o sistema de saúde em relação ao aumento dos custos, a capacitação dos recursos humanos, investimentos em infraestrutura física e as necessidades de atualização dos instrumentos de regulação e certificação (BRASIL, 2009).

A partir desse cenário atual de crise econômica, social e política, tanto no âmbito nacional como no internacional, o Brasil necessita urgentemente superar barreiras na consolidação do direito a saúde no país. Primeiramente é notório destacar que para responder a esta crise e defender o direito a saúde é indispensável analisar e relembrar o nascimento do Sistema Único de Saúde (SUS) fundamentado na constituição de 1988, entender o contexto atual e compreender as tendências que se projetam. Desta forma, a avaliação da qualidade de serviços de saúde se estabelece como uma boa estratégia de apoio a gestão e controle social, tudo isso fundamentado pelas diretrizes básicas do SUS como a descentralização, integralidade e participação social (SOUZA LEPF, et al., 2019).

Em suma, avaliar pode fornecer dados tanto para a melhoria das intervenções em saúde como para o julgamento a respeito de sua cobertura, acesso, equidade, qualidade, efetividade, eficiência e perspectivas dos usuários a seu respeito (HARTZ ZMDA e VIEIRA-DA-SILVA LM, 2005).

Há uma grande carga mórbida neste grupo populacional que são os portadores de doenças crônicas, que por vezes são sub diagnosticados, em decorrência de prováveis falhas na assistência. Além disso, a possibilidade de que pessoas com maior risco para complicações e incapacidades não estejam sendo priorizados na oferta de ações as suas necessidades, uma vez que os serviços de saúde ainda elegem o planejamento da oferta em detrimento das necessidades de atenção existentes (SOUZA MFM, et al., 2018)

Diante do cenário de alterações ocorridas no mundo atualmente e com o crescimento populacional do número de idosos, onde as doenças crônicas não transmissíveis (DCNT) continuam liderando como a principal causa de morte, ainda assim no Brasil após os 30 anos da implantação do SUS, observa-se uma 
redução na taxa de mortalidade por doenças cardiovasculares, por outro lado um aumento na taxa de mortalidade por diabetes (SOUZA MFM, et al., 2018). A provisão de cuidados a pessoa com condição crônica torna-se urgente no sentido da sua execução, contudo é uma tarefa complexa, envolvendo um enfoque teórico que proporcione a reelaboração das práticas de cuidado a luz da reflexão do dia a dia do serviço (BRASIL, 2011).

Embora os debates estejam acontecendo, ainda são limitados os estudos na literatura referentes a esta temática Assistência à saúde prestada aos usuários hipertensos e diabéticos no Brasil o que motiva a realização deste estudo. Destaca-se que a busca pela qualidade dos serviços de assistência em saúde no Brasil tem recebido grande interesse do Ministério da Saúde (MS), com ênfase para a atenção ao indivíduo idoso com doenças crônicas, haja vista a sua relevância na adequação das políticas públicas as necessidades desse grupo etário. Considera-se que a detecção precoce, acompanhamento e controle, reforçado por tecnologias de atenção à saúde são as principais estratégias para prevenir e/ou postergar o início e a evolução das complicações e das incapacidades decorrentes da HAS e DM (BRASIL, 2011).

Isto posto, o artigo contribui para a construção do conhecimento relacionado a assistência prestada pelas equipes de saúde da família, bem como colabora para a análise e a reorganização das práticas assistenciais a pessoa com HAS e DM na rede de atenção, a luz da identificação de problemas e da compreensão dos determinantes facilitadores e limitadores dessas práticas as quais poderão ser identificadas como norteadores de ações de melhoria. Diante disso, o artigo tem como objetivo analisar as evidências disponíveis na literatura cientifica sobre a assistência à saúde prestada aos usuários hipertensos e diabéticos no Brasil.

\section{MÉTODOS}

Trata-se de um estudo de revisão integrativa, onde o interesse foi ter um cenário nacional da produção científica em periódicos brasileiros sobre a avaliação da assistência à saúde prestada aos usuários portadores de HAS e DM, no período de 2009 a 2018, sendo esse período escolhido por compreender a substituição do sistema HIPERDIA pelo sistema e-SUS da Atenção Básica (AB) pelo Ministério da Saúde por meio da Coordenação Geral de Atenção as Pessoas com Doenças Crônicas (2013), pautado pela Política Nacional de Atenção Básica (PNAB), pelo Programa Nacional de Melhoria da Qualidade (PMAQ) e pela Política Nacional de Informática e Informação em Saúde (PNIIS). O que provocou uma série de mudanças no acompanhamento e controle acerca da assistência à saúde dos hipertensos e diabéticos brasileiros.

Como o interesse foi estudar a realidade do cenário nacional, foram escolhidos artigos brasileiros, publicados em periódicos indexados nas bases de dados Literatura Latino Americana e do Caribe em Ciências da Saúde (LILACS), Banco de dados em Enfermagem (BDENF), Scientific Eletronic Libary Online (Scielo). A escolha por essas bases de dados deveu-se pela abrangência e importância ao reunir número elevado de periódicos nacionais da saúde.

A partir da escolha das bases de dados da pesquisa, foram selecionados os descritores mais apropriados para a busca dos artigos, atentando para os critérios de inclusão e exclusão. Após ter encontrados os descritores para acessar a busca, pôde-se combiná-los entre si utilizando o operador boleano AND.

Para a seleção dos artigos, foram definidos os seguintes critérios de inclusão: 1) idioma: português, espanhol e inglês; 2) ter sido publicado no período entre 2009 e 2018 e; 3) tratar de temas relacionados com assistência â hipertensão e diabetes no Brasil. Também foi formulado como critério de exclusão: 1) não se tratar de artigo empírico como revisão de literatura, relatórios e editorial; 2) tratar de outros temas que não se relacionem com assistência a hipertensão e diabetes e; 3 ) artigos duplicados, publicados em mais de uma base de dados. As referências utilizadas na introdução tiveram papel relevante de apresentação da temática do estudo.

\section{RESULTADOS}

A Figura 1 ilustra o processo que foi realizado para reunir os artigos usados para a presente revisão integrativa, sendo guiada pelos descritores supracitados. Logo, ao final do procedimento restou 9 estudos exclusivos para análise. 
Figura 1 - Fluxograma de seleção dos estudos.

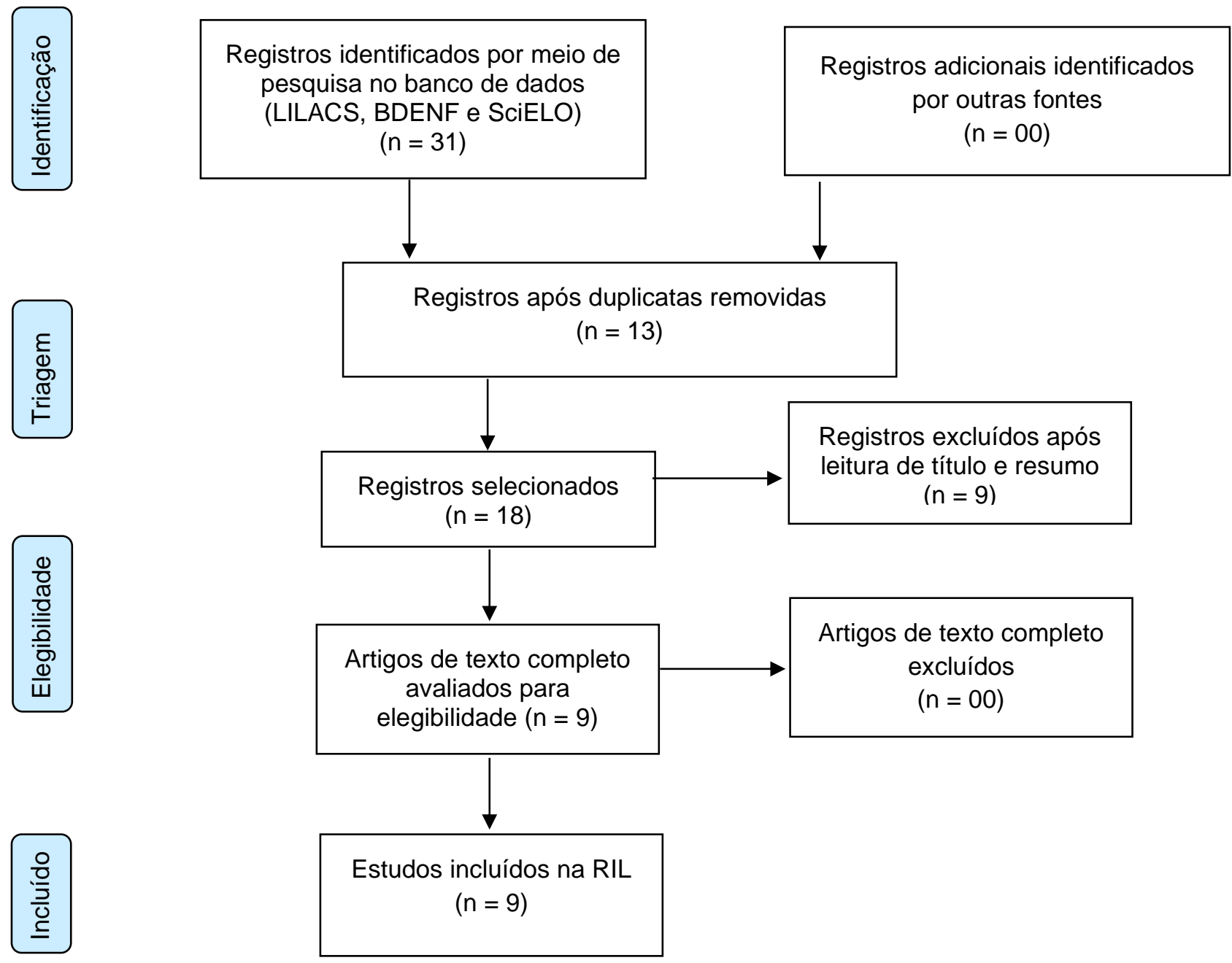

Fonte: Ferreira FC e Silva MVS, 2021.

Os 9 artigos selecionados foram escritos em língua portuguesa e publicados em periódicos brasileiros. No período avaliado, o único ano que não publicaram estudos sobre o tema foi o ano de 2018, já em 2014 foram publicados dois artigos, contudo os anos 2010, 2011, 2012, 2013, 2015, 2016 e 2017 tiveram um artigo cada.

Destaca-se que dos nove estudos publicados sobre o tema avaliação da assistência aos hipertensos e diabéticos, sete foram artigos e dois foram dissertações de mestrado. Quanto à classificação de periódicos Qualis da Capes, identifica-se que os estudos foram publicados em revistas muito bem classificadas, sendo dois periódicos classificados como B1, três periódicos B2 e um periódico B4. Já a avaliação da Capes para o Programa de pós-graduação em Enfermagem da Universidade Federal do Paraná (UFPR) Conceito 5, e para o Programa de Pós-graduação em Saúde Pública, Escola Nacional de Saúde Pública Sérgio Arouca, Rio de Janeiro. Conceito 6 . Isto é, muito bem avaliados. A grande maioria dos estudos teve delineamento quantitativo $(n=7)$, método misto $(n=1)$ e qualitativo $(n=1)$.

Com respeito às amostras estudadas, dessas nove pesquisas verificou-se o predomínio de cinco estudos com usuários hipertensos e ou diabéticos em suas amostras, um com profissionais e usuários, um com análise documental das fichas de cadastros, um artigo comparou duas unidades básicas de saúde e, por fim uma pesquisa trabalhou como amostra as unidades de saúde e domicílios.

O Quadro 1 sintetiza as principais informações dos artigos selecionados, a saber: autores/ano e os resultados mais relevantes. 
Quadro 1 - Síntese dos principais achados sobre determinado tema.

\begin{tabular}{|c|c|c|c|}
\hline $\mathbf{N}$ & Autores (Ano) & Periódico & Principais achados \\
\hline 1 & $\begin{array}{l}\text { Carvalho Filha FSS, et al. } \\
\qquad(2011) .\end{array}$ & Rev Rene, 12 (n. esp.): 930-936. & $\begin{array}{l}\text { Estudo descritivo, avaliativo com abordagem quantitativa, realizado nas } \\
\text { unidades básicas de saúde de caxias no Maranhão, e com uma amostra de } \\
1024 \text { sujeitos. Dentre os achados observou-se diversos entraves na aplicação } \\
\text { das ações de controle da hipertensão e diabetes que vão desde a falta de } \\
\text { materiais e equipamentos essenciais para o controle e tratamento, como por } \\
\text { exemplo os medicamentos e glicosímetro até à não execução de atividades de } \\
\text { educação em saúde pelos profissionais da equipe saúde da família. }\end{array}$ \\
\hline 2 & Aleluia IRS, et al. (2017). & Ciências e Saúde Coletiva, 22(6): 1845-1856. & $\begin{array}{l}\text { Estudo de caso em um municipio sede da região nordeste do estado da Bahia, } \\
\text { com dois niveis de analise: a equipe professional e a gestão municipal. } \\
\text { Identificou-se que coordenação do cuidado na atenção primária a saúde } \\
\text { apresenta dificuldades, destacando-se como as principais: a ausência de } \\
\text { protocolos assistências, de sistemas informatizados, e de outras tecnologias de } \\
\text { informação e comunicação. }\end{array}$ \\
\hline 3 & Correia LOS, et al. (2014). & Ciências e Saúde Coletiva, 19(6): 1685-1697. & $\begin{array}{l}\text { Estudo descritivo, observacional e transversal, a amostra de } 287 \text { fichas } \\
\text { cadastradas e obtida de forma aleatoria e proporcionalmente entre } 5 \text { regiões do } \\
\text { estado de Alagoas.Em quase } 70 \% \text { dos registros de dados do cadastro sistema } \\
\text { Hiperdia apresentou boa completitude. Todavia foi encontrada deficiências na } \\
\text { completitude no preenchimento de campos obrigatórios como numeral do } \\
\text { logradouro nos dados referentes a identificação, em algumas informações } \\
\text { referentes aos responsáveis pelo atendimento, 35\% dos cadastros realizados o } \\
\text { responsável pelo atendimento não assinou a ficha. }\end{array}$ \\
\hline 4 & $\begin{array}{l}\text { Carvalho Filha FSS, et al. } \\
(2014) .\end{array}$ & Saúde Debate. Out., 389 (n. esp.):265-268. & $\begin{array}{l}\text { Pesquisa avaliativa com tres eixos de investigação, utilizando a triangulação de } \\
\text { método, sendo realizado em caxias no Maranhão, com } 120 \text { profissionais e } 1024 \\
\text { usuarios da atenção básica. Notou-se que os profissionais ao avaliarem o plano } \\
\text { de reorganização da atenção a hipertensão e diabetes são divergentes quanto } \\
\text { aos pontos positivos e negativos do referido plano, como por exemplo ao } \\
\text { citarem os medicamentos, em um dado momento elogiaram a distribuição } \\
\text { gratuita e em outro informaram que a falta constante e as dificuldades de acesso } \\
\text { são pontos negativos. Quanto aos usuários, } 64,1 \% \text { avaliaram assistência } \\
\text { recebida como boa. }\end{array}$ \\
\hline 5 & Radigonda B, et al. (2015). & Saúde Debate, 38 (105): 423-431. & $\begin{array}{l}\text { Estudo avaliativo de base normativa com } 687 \text { individuos hipertensos e } \\
\text { diabeticos. Observou-se que a proporção de pessoas com hipertensão e } \\
\text { diabetes representam } 63,1 \% \text { e } 62,8 \% \text { respectivamente, essas proporções } \\
\text { indicam que a detecção tanto das pessoas com hipertensão quanto com } \\
\text { diabetes pelas equipes de saúde da família das UBS é regular, sendo que a } \\
\text { meta a sugerida pelo MS é de } 100 \% \text {. }\end{array}$ \\
\hline
\end{tabular}




\begin{tabular}{|c|c|c|c|}
\hline $\mathbf{N}$ & Autores (Ano) & Periódico & Principais achados \\
\hline 6 & Radigonda B, et al. (2016). & Epidemiol. Serv. Saúde, 25 (1): 115-126. & $\begin{array}{l}\text { Esudo avaliativo de base normativa, realizado em cambé no Paraná, com } 668 \\
\text { idosos hipertensos e/ou diabéticos atendidos na atenção básica. Verificou-se } \\
\text { que a cobertura assistencial de consultas médicas ( } 69,2 \%) \text { está classificada } \\
\text { como regular, as consultas de enfermagem ( } 1,5 \% \text { e as visitas domiciliares } \\
\text { ( } 12,2 \%) \text { classificadas como críticas, no que diz respeito a concentração de } \\
\text { consultas medicas, de enfermagem e visitas domiciliares aos grupos de alto } \\
\text { risco também apresentam uma classificação crítica. }\end{array}$ \\
\hline 7 & $\begin{array}{l}\text { Domini Filho LA, et. al. } \\
\text { (2010). }\end{array}$ & Bras. Clin. Méd., 8(6): 509-512 & $\begin{array}{l}\text { Estudo avaliativo e observacional sendo realizado em rincão no estado de São } \\
\text { Paulo com uma amostra de } 147 \text { sujeitos usuarios de uma unidade basica de } \\
\text { saúde no "projeto saúde e vida". Observou-se que a terapêutica não } \\
\text { farmacológica é uma alternativa para o controle da Hipertensão e diabetes na } \\
\text { atenção primária a saúde. } 74 \text { participantes portadores de diabetes mellitus e } \\
\text { hipertensão arterial sistêmica foram seguidos no período de abril de } 2005 \text { a } \\
\text { junho de } 2006 \text {, dos quais } 36 \text { frequentaram mais de } 5 \text { encontros, onde se discutia } \\
\text { assuntos relacionados a hipertensão e diabetes, realizavam mensuração } \\
\text { antropométricas, e se encontravam } 2 \text { vezes por semana para realização de } \\
\text { atividade física, havendo melhora pressórica em } 25 \text { (69,4\%) pacientes e } \\
\text { melhora glicêmica em } 24 \text { (66.7\%) pacientes. Entre os com menos de } 5 \\
\text { encontros (38 pacientes), destes } 19(55,9 \%) \text { tiveram melhora pressórica e } 15 \\
(54,29 \%) \text { melhora glicêmica. }\end{array}$ \\
\hline 8 & Mendes LVP (2013). & Escola de Saúde Pública Sérgio Arouca. [Dissertação] & $\begin{array}{l}\text { Estudo transversal e observacional utilizando base de dados secundário, } \\
\text { realizado no estado do Rio de Janeiro com uma amostra de } 547 \text { domicilios. } \\
\text { Verificou-se que com a adesão ao tratamento medicamentoso, a } \\
\text { automedicação e os cuidados com medicamentos como forma do Uso Racional } \\
\text { de Medicamentos (URM) tem baixa adesão entre os hipertensos e diabéticos, } \\
\text { sendo maior entre os hipertensos casados, que possuem ocupação } \\
\text { remunerada, que referem receber orientações de seu médico. }\end{array}$ \\
\hline 9 & Silva JVM (2012). & Universidade Federal do Paraná. [Dissertação]. & $\begin{array}{l}\text { Pesquisa qualitativa, realizada em Colombo região metropolitanaa de curitiba, } \\
\text { sendo os dados coletados por meio de uma entrevista semiestruturada e } \\
\text { gravada com } 30 \text { usuarios da atenção básica. Observou-se que a descoberta da } \\
\text { doença, o motivo para a busca do serviço, o conhecimento do programa e } \\
\text { avaliação do programa são fatores que interferem na adesão, acompanhamento } \\
\text { e controle ao tratamento. Após análise dos estudos verificou-se que os usuários } \\
\text { identificaram a descoberta da doença como o ponto de partida para a adesão } \\
\text { ao programa de hipertensão e diabetes. }\end{array}$ \\
\hline
\end{tabular}

Fonte: Ferreira FC e Silva MVS (2021). 


\section{DISCUSSÃO}

O presente estudo expôs a incipiência na utilização do plano de ações estratégicas para o enfrentamento das doenças crônicas não transmissíveis (DCNT) no Brasil 2011-2022 como norteador das ações direcionadas ao cuidado à pessoa que vivencia uma condição crônica de saúde. O plano é recomendado para reorganizar a rede de atenção à saúde no intuito de aperfeiçoar a assistência prestada por profissionais atuantes na estratégia saúde da família (ESF).

Sobre a avaliação da estrutura onde são desenvolvidas a assistência de saúde, o presente estudo identificou que existe vários entraves na organização estrutural do serviço de saúde, que vai desde a falta de materiais e equipamentos até protocolos assistenciais necessários para o desenvolvimento das ações de atenção à saúde.

A análise da estrutura acontece em nível institucional e compreende também a avaliação dos materiais, ou seja, avaliam-se a área física, os recursos humanos, materiais e financeiros adicionando a capacitação profissional e a organização dos serviços ou ações de saúde (PERTENCE PP, 2010).

Pode-se perceber que as avaliações das estruturas das unidades de saúde na atenção primária apresentam múltiplas fragilidades em relação a estrutura organizacional, dentre elas estão: estrutura física inadequada, falta de materiais e equipamentos como medicamentos, glicosímetro, balanças antropométricas, entre outros, até a ausência de protocolos assistenciais padrões, sistemas de informação e registros documentais incompletos (CARVALHO FILHA, et al., 2011; ALELUIA IRS, et al., 2017; CORREIA LOS, et al., 2014 e CARVALHO FILHA FSS, et al., 2014).

Perante o exposto, a falta ou a dificuldade de acesso aos medicamentos incorre no impedimento da adesão ao tratamento, a inexistência do glicosímetro, de balança antropométrica ou de qualquer outro material de mensuração implicam na dificuldade no acompanhamento e controle, a falha ou ausência de protocolos assistências padrões provoca um atendimento ineficaz, fichas cadastrais com registros incompletos como por exemplo a ausência do numeral do logradouro incompatibiliza a busca ativa ou detecção de casos novos ou até mesmo no acompanhamento e controle. Com isso, de um modo geral o serviço relacionado a estrutura apresenta-se de forma inadequada para assistência a atenção aos portadores de hipertensão e diabetes nas unidades da atenção primaria à saúde.

Desta forma, os dados relacionados a protocolos assistenciais encontrado pelo presente estudo se assemelham aos achados na literatura, o qual observou que existem estratégias recomendadas na implementação de práticas de saúde baseadas em evidencias, assim como o acompanhamento dessas intervenções. Embora exista elevado nível de consonância sobre as melhores práticas de prevenção, diagnóstico e tratamento de diabetes e hipertensão arterial, descrita em diversos protocolos assistenciais propostos pelo MS e na literatura, o seu uso ainda é incipiente, principalmente quando se trata de implementação e registro de ações junto a população acompanhada, transformando-se em obstáculos para o alcance de melhores resultados assistenciais fundamentado nas evidencias cientificas atuais (SILVA LB, 2018).

Além disso, essa lacuna poderá ser amenizada pelo gestor da coordenação de cuidados e pelos profissionais de saúde da equipe que ao se reunirem para planejamento, elaborem protocolos assistenciais que levem em consideração as evidencias cientificas no que diz respeito as práticas de saúde dando importância a realidade da família e da comunidade.

A qualidade da atenção deve ser avaliada pela associação dos serviços e aplicação dos recursos disponíveis, para obter os melhores índices de saúde dos usuários do sistema, ao mais baixo custo de recursos e com os mais baixos riscos e efeitos adversos sobre o indivíduo, a comunidade e o sistema (STEIN AT, 2005).

Assim sendo, os achados encontrados na pesquisa estão de acordo com estudos encontrados na literatura, que constatam a insatisfação das equipes e dos usuários quanto a estrutura organizacional das unidades básicas de saúde. Já o estudo realizado em Cuiabá de 2009 a 2013, apresentou um resultado 
contrário ao atual estudo, que evidenciou que $62 \%$ das equipes de saúde classificaram como aceitável a estrutura organizacional das unidades básicas de saúde (UBS) (CAPUCHINHO PFM, et al., 2015 e PORTELA LR, et al., 2013).

Estes resultados encontrados pelo presente estudo se assemelham aos encontrados por outros autores, onde evidenciaram a alta insatisfação dos usuários com as condições oferecidas, o que implica em condições das unidades de saúde insatisfatória, condições que são fundamentais para a implementação das ações e que permitem a consolidação da ESF. Apesar da ESF demonstrar uma evolução na efetividade e qualidade dos serviços prestados na APS, constata-se um número significativo de unidades de saúde com estrutura inadequada, não raro, improvisada, onde a carência de equipamentos e insumos, restringem o escopo das ações e resolubilidade, limitando a capacidade de resposta aos problemas de saúde do indivíduo e da comunidade (SANTIAGO RF, et al., 2013 e BOUSQUAT A, et al., 2017).

O manual de estrutura das UBS destaca que para solucionar pelo menos cerca de $85 \%$ das dificuldades ou entraves da assistência à saúde do indivíduo e/ou da comunidade, são necessários dispor de recursos estruturais e equipamentos adequados que proporcionem a ação dos profissionais de saúde. Assim a estrutura organizacional contribui como facilitadora das práticas de saúde das equipes de saúde da família, consolidando o novo modelo de atenção proposto pelo SUS (BRASIL, 2008).

A relação entre estrutura e qualidade da atenção é de suma importância para o planejamento e organização dos sistemas ou dos serviços, além de que o objetivo é possibilitar serviços ou ações de saúde que melhorem a qualidade da atenção aos usuários (DONABEDIAN A, 1993).

Desta forma, a estrutura corresponde a um elemento primordial para a organização da APS mais efetiva. Por isso, existe a necessidade constante de avaliação, com intuito de captar investimentos para a estrutura da área física e na aquisição de equipamentos de mensuração ou até mesmo de sistemas de informação que facilitem a comunicação, ou seja, que assegurem a resolubilidade da atenção à saúde do portador de hipertensão e diabetes, melhorando a efetividade dos serviços.

Assim como, a avaliação do processo de atendimento é relevante e contribui com a elevação da qualidade dos cuidados prestados aos usuários através da organização do processo de trabalho das equipes de saúde da família, estruturando e consolidando a APS.

As ações de detecção e acompanhamento tanto das pessoas portadoras de hipertensão como diabetes monitoradas pelas equipes de saúde da família das UBS é regular. $E$ isto, está relacionado com a dificuldade que as equipes possuem de seguir as recomendações da operacionalização das ações de detecção, acompanhamento e controle, ou seja, de seguir as orientações preconizadas pelo plano de reorganização da atenção à hipertensão arterial e diabetes mellitus (RADIGONDA B, et al., 2015, RADIGONDA B, et al., 2016 e SILVA JVM, 2012).

O reconhecimento das inadequacidades na assistência, mostra que as equipes seguem de forma parcial as orientações preconizadas pelo MS, contudo existem achados que confirmam que os profissionais estão desempenhando suas funções em um sistema público com recursos humanos, físicos e de materiais insuficientes, decorrente do baixo investimento financeiro no campo da saúde, e da falta de compromisso dos políticos. Logo, os serviços são de baixa efetividade, devido aos baixos investimentos financeiros nas equipes e principalmente pela falta de compromisso dos gestores (SILVA LB, 2018 e DOMINI FILHO, et al., 2010).

Identifica-se outras causas para baixa efetividade da assistência, como a baixa cobertura assistencial de consultas médicas e de enfermagem, ou seja, uma cobertura assistencial de consultas medicas regular $(69,2 \%)$ e de enfermagem crítica (1,5\%) (SILVA LB, 2018).

Ainda sobre as consultas, os achados evidenciados no presente estudo são preocupantes visto que suas porcentagens podem ser subestimadas, em decorrência dos subregistros das atividades realizadas nas UBS, principalmente relacionadas a enfermagem, também identificados neste estudo.

Diante da realidade apresentada, recomenda-se delineamento de outros estudos que apontem os reais motivos que levam os prováveis subregistros do processo de trabalho das equipes de saúde da família especialmente a enfermagem, de tal forma a subsidiar caminhos alternativos para a superação desses 
desafios. Ressalta-se que o registro em prontuário potencializa o desenvolvimento e monitoramento de vários indicadores de avaliações qualitativas e quantitativas, as quais poderão trazer muitos benefícios a assistência à saúde, dentre estes, o melhoramento de recursos com impacto na resolubilidade.

Também foi evidenciado no presente estudo que as ações de detecção e acompanhamento são maiores em mulheres, em pessoas iguais ou acima de 60 anos, com baixa escolaridade, sem atividade remunerada e que integra a camada economicamente mais baixa da sociedade. Dados semelhantes foram encontrados em estudo realizado no município de Teixeira em Minas Gerais em 2009 no qual foi observado a predominância de pessoas com perfil semelhante. Logo, esses indicadores são uteis para o planejamento das ações a serem implementadas por gestores da coordenação de cuidados e profissionais das equipes de ESF na assistência aos hipertensos e diabéticos (MENDES LVP, 2013).

Isto posto, os achados encontrados pelo presente estudo estão de acordo com a literatura, onde afirmam que, a ausência de padronização das ações significa fragilidade de gestão e consequentemente de cuidados prestados, podendo levar a uma variação nos modos de fazer. Por isso, é desejável o comprometimento de gestores e profissionais assistenciais no sentido de se construir um processo de trabalho pautado nas ações preconizadas pelo plano de reorganização da atenção a hipertensão arterial e diabetes mellitus, baseado no rastreamento, capacitação profissional e reorganização da rede de atenção à saúde, sendo planejado e implementado pela equipe, respeitando o indivíduo e as particularidades regionais da comunidade, buscando com isso efetividade na operacionalização das ações de saúde (WERNECK MAF, et al., 2009).

O controle da hipertensão e diabetes tem se mostrado eficaz na redução de complicações destas doenças. Contudo não há evidencia cientifica incontestável a respeito da eficiência dos programas de rastreamentos. Essas ações de detecção, acompanhamento e controle são boas oportunidades de se coletarem dados observacionais que apesar de não substituir a evidencia direta de ensaios clínicos randomizados, podem gerar relevantes evidencias sobre eficiência, custo e impacto (TOSCANO CM, 2004).

Desta forma, os estudos apontam que as ações de rastreamento são eficazes na redução das complicações da hipertensão e diabetes, contudo as equipes de saúde da família encontram dificuldades na execução das ações pois muitas equipes não seguem ou seguem parcialmente as orientações do plano de reorganização da atenção a hipertensão arterial e diabetes mellitus, e estas equipes trabalham com baixos investimentos financeiros e com poucos gestores compromissados com o serviço. Diante disso, as práticas assistenciais mantem-se distanciadas das atuais propostas de se construir uma rede de atenção as condições crônicas que requerem cuidados integrado, longitudinal, proativo e inovador.

Como limitações deste estudo destaca-se a inclusão de inúmeros estudos com diferentes delineamentos de pesquisas, o que complicou um pouco a análise dos estudos, dificultando estabelecer relação da estrutura e processo da avaliação de donabedian com os parâmetros assistenciais e a qualidade dos cuidados de saúde na atenção a hipertensão e diabetes.

Apesar da fragilidade supracitada, o estudo apresenta potencialidades. Dentre elas, a variedade do processo de amostragem que proporcionou um aprofundamento e abrangência das conclusões da revisão, consequentemente, contribuindo com um retrato compreensivo da qualidade da assistência prestada aos hipertensos e diabéticos no Brasil. Outro ponto de grande potencial diz respeito a escolha do método de pesquisa pois foi possível obter um profundo entendimento da qualidade da assistência prestada aos hipertensos e diabéticos baseando-se em estudos anteriores.

\section{CONSIDERAÇÕES FINAIS}

O estudo mostrou baixa qualidade nas práticas assistenciais a estas morbidades, dentre as causas estão os baixos investimentos na assistência. A lacuna encontrada neste estudo diz respeito ao subregistros das atividades das equipes e como isto impacta na saúde do indivíduo. As evidências obtidas neste artigo sugerem que a maior efetividade das práticas assistenciais, se sustentam quando a gestão do serviço assistencial faz investimentos na estrutura das UBS e estimulam a capacitação e a construção de processos de trabalho baseado na demanda da comunidade. Além disso, os achados deste artigo subsidiarão a atuação dos gestores e profissionais, articulando melhoria do processo de trabalho na ESF por meio da atuação de uma equipe multidisciplinar, comprometida com uma asistência equitativa, integral e resolutiva. 


\section{REFERÊNCIAS}

1. ALELUIA IRS, et al. Coordenação do cuidado na atenção primária à saúde: estudo avaliativo em um município sede de macrorregião do nordeste brasileiro. Ciências e Saúde coletiva. 2011; 22(6):1845-1856.

2. BOUSQUAT A, et al. Tipologia da estrutura das unidades básicas de saúde brasileiras: Os 5 R. Cad. Saúde pública. 2017. 33(8).

3. BRASIL. Manual do Ministério da Saúde. 2008. Manual de estrutura fisica das unidades básicas de saúde: Saúde da família. Disponivel em: bvsms.saude.gov.br. Acessado em: 20 de Agosto de 2019.

4. BRASIL. Manual do Ministério da Saúde. 2009. Avaliação de tecnologias em saúde: ferramentas para gestão do SUS. Disponivel em: bvsms.saude.gov.br. Acessado em: 22 de Agosto de 2019.

5. BRASIL. Manual do Ministério da Saúde. 2011. Plano de ações estratégicas para o enfrentamento das doenças crônicas não transmissiveis (DCNT) no Brasil. Disponivel em: bvsms.saude.gov.br. Acessado em: 20 de Agosto de 2020.

6. CAPUCHINHO PFM, et al. Infraestrutura, equipamentos, insumos, imunobiológicos e medicamentos: avaliação da dimensão da unidade básica da saúde Im: Anais do fórum de ensino, pesquisa, extensão e gestão; 2015; Montes Claros.

7. CARVALHO FILHA FSS, et al. Avaliação do controle de hipertensão e diabetes na atenção básica: perspectiva de profissionais e usuários. Saúde debate. 2014 out; 389n. esp.):265-278.

8. CARVALHO FILHA FSS, et al. Hiperdia: adesão e percepção de usuários acompanhados pela estratégia saúde da família. Ver Rene. 2011; 12(n. esp.):930-936.

9. CORREIA LOS, et al. Completitude dos dados cadastrados de portadores de hipertensão arterial e diabetes mellitus registrados no sistema hiperdia em um estado do nordeste do Brasil. Ciências e Saúde coletiva. 2014; 19(6):16851697.

10. DOMINI FILHO LA, et al. Impacto de um modelo de tratamento não farmacológico para diabetes e hipertensão no município de rincão: projeto saúde e vida. Ver Bras Clin Méd. 2010; 8(6):509-512.

11. DONABEDIAN A. Prioridades para el progresso em la evaluación y monitoreo de la atención. Salud Pública de México. 1993; 35(1):94-97.

12. FIGUEIREDO JR AM, et al. Prevalência de sobrepeso, obesidade e alterações de pressão arterial em crianças do ensino fundamental de uma escola privada em Belém-PA. Revista Eletrônica Acervo Saúde, 2019; 35: e1691.

13. HARTZ ZMDA, VIEIRA-DA-SILVA LM. Avaliação em saúde: dos modelos teóricos à prática na avaliação de programas e sistemas de saúde. Salvador, Rio de Janeiro: Edufba, Editora FIOCRUZ, 2005. f.275.

14. HU JR CJ. The public health dimensiono f chronic kidney disease: what we have learnt over the past decade. Nephol dial transplant. 2017; 32 (suppl):113-120.

15. MENDES LVP. Desempenho da atenção primária e uso racional de medicamentos por pacientes hipertensos e diabéticos no municipio do Rio de Janeiro [dissertação] Rio de Janeiro: Escola de Saúde Pública Sérgio Arouca, 2013, $115 p$.

16. PERTENCE PP e MELLEIRO MM. Implantação de ferramenta de gestão de qualidade em hospital universitário. Revista da Escola de Enfermagem da USP. 2010; 44(4):1024-1031.

17. PORTELA LR, et al. Programa nacional da melhoria do acesso de qualidade da atenção básica: análise da autoavaliação em Sobral, Ceará, Sonare 2013: 12(1):40-5.

18. RADIGONDA B, et al. Avaliação da cobertura da atenção básica na detecção de aadultos com diabetes e hipertensão. Saúde debate. 2015; 38(105):423-431.

19. RADIGONDA B, et al. Avaliação do acompanhamento de pacientes adultos com hipertensão e /ou diabetes melito pela estratégia saúde da família e identificação de fatores associados, cambé-PR, 2012. Epidemiol. Serv. Saúde. 2016; 25(1):115-126.

20. SANTIAGO RF, et al. Qualidade do atendimento nas unidades de saúde da unidade da família do município de Recife: a percepção dos usuários. Ciências e Saúde coletiva. 2013; 18(1):35-44.

21. SILVA JVM. O enfermeiro e a avaliação do usuário sobre o atendimento do programa de hipertensão arterial e diabete mellitus [dissertação]. Setor de Ciências da Saúde, Universidade federal do Paraná. 2012. 88f.

22. SILVA LB. Qualidade do cuidado à pessoa idosa com diabetes e/ou hipertensão atendida na atenção primária à saúde [dissertação]. Belo Horizonte: Universidade Federal de Minas Gerais; 2018. $216 f$.

23. SOUZA LEPF, et al. Os desafios atuais da luta pelo direito universal à saúde no Brasil. Ciências e Saúde Coletiva, 2019, 24(8): 2783-2792.

24. SOUZA MFM, et al. Transição da saúde e da doença no Brasil e nas unidades federadas durante 30 anos do SUS. Ciências e Saúde Coletiva, 2018, 23(6):1737-1750.

25. STEIN AT. Busca da melhor evidência e de efetividade no GHC. Momento e perspectiva saúde. Revista técnico cientifica do grupo hospitalar conceição. 2005; 18(2):72-73.

26. TAO L, et al. Tendency for age-especific mortality with hypertension in the european union fron 1980 to 2011 . Int $\mathrm{J}$ Clin Exp Med. 2015; 8(2): 1611-1623.

27. TOSCANO CM. As campanhas nacionais para detecção das doenças crônicas não transmissíveis: diabetes e hipertensão arterial. Ciência e Saúde coletiva. 2004; 9(4):885-895.

28. WERNECK MAF, et al. Protocolos de cuidados à saúde e organização do serviço. Nescon, Universidade Federal de Minas Gerais. 2009; 82f. 